\title{
Early Career Professionals' (Researchers, Practitioners, and Policymakers) Role in Advocating, Disseminating, and Implementing the Global Action Plan on Physical Activity: ISPAH Early Career Network View
}

\author{
Artur Direito, Joseph J. Murphy, Matthew Mclaughlin, Jacqueline Mair, Kelly Mackenzie, \\ Masamitsu Kamada, Rachel Sutherland, Shannon Montgomery, and Trevor Shilton, on behalf of the \\ ISPAH Early Career Network
}

\begin{abstract}
Increasing population levels of physical activity (PA) can assist in achieving the United Nations sustainable development goals, benefiting multiple sectors and contributing to global prosperity. Practices and policies to increase PA levels exist at the subnational, national, and international levels. In 2018, the World Health Organization launched the first Global Action Plan on Physical Activity (GAPPA). The GAPPA provides guidance through a framework of effective and feasible policy actions for increasing PA, and requires engagement and advocacy from a wide spectrum of stakeholders for successful implementation of the proposed actions. Early career professionals, including researchers, practitioners, and policymakers, can play a major role with helping "all people being regularly active" by contributing to 4 overarching areas: (1) generation-of evidence, (2) dissemination —of key messages and evidence, (3) implementation —of the evidence-based actions proposed in the GAPPA, and (4) contributing to advocacy for robust national action plans on PA. The contribution of early career professionals can be achieved through 5 pathways: (1) research, (2) workplace/practice, (3) business, (4) policy, and (5) professional and public opinion. Recommendations of how early career professionals can contribute to the generation, dissemination, and implementation of the evidence and actions proposed by the GAPPA are provided.
\end{abstract}

Keywords: policy, public health, public health practice, advocacy

Insufficient physical activity (PA) is a key risk factor for noncommunicable diseases, morbidity, and mortality globally, ${ }^{1}$ leading to large health care costs and productivity losses. ${ }^{2}$ Despite the wealth of research on effective interventions (eg, mass media campaigns, urban design, social support (for PA) in workplaces and communities $)^{3}$ and existing PA policies and plans, ${ }^{4}$ global PA levels are not improving. ${ }^{5}$ Furthermore, the prevalence of insufficient PA is estimated to be twice as high in high-income countries compared with low-income countries (36.8\% vs $16.2 \%$ ), which is important given the fast transitions of low onto middle-/high-income econo-

Direito is with the Division of Endocrinology, Department of Medicine, Yong Loo Lin School of Medicine, National University of Singapore, Singapore. Murphy is with the Department of Physical Education and Sport Sciences, University of Limerick, Limerick, Ireland. Mclaughlin is with the School of Medicine and Public Health, The University of Newcastle, Callaghan, NSW, Australia; Hunter New England Population Health, Wallsend, NSW, Australia; Hunter Medical Research Institute, New Lambton, NSW, Australia; and the Priority Research Centre for Health Behaviour, The University of Newcastle, Callaghan, NSW, Australia. Mair is with the School of Applied Sciences, Edinburgh Napier University, Edinburgh, Scotland, United Kingdom. Mackenzie is with the School of Health and Related Research, University of Sheffield, Sheffield, United Kingdom. Kamada is with the Department of Health and Social Behavior, School of Public Health, Graduate School of Medicine, The University of Tokyo, Bunkyo, Tokyo, Japan. Sutherland is with Hunter New England Population Health, The University of Newcastle, Callaghan, NSW, Australia. Montgomery is with the Centre for Public Health, School of Medicine, Dentistry and Biomedical Sciences, Queen's University Belfast, Belfast, United Kingdom. Shilton is with Cardiovascular Health, National Heart Foundation of Australia, Melbourne, VIC, Australia. Direito (artur.direito@ nus.edu.sg) is corresponding author. mies and corresponding urbanization and increase in sedentary occupations, leading to possible declines in PA. ${ }^{6,7}$

Although an abundance of information regarding the benefits, recommendations, and promotion of PA is available, global efforts to increase PA have been unsatisfactory. ${ }^{5}$ There is a clear need to make better use of the available evidence and mobilize advocacy to successfully translate knowledge into practice and policy, ${ }^{8}$ avoiding research waste and ultimately improving health. ${ }^{9}$ Practices and policies to increase population levels of PA exist but need to be prioritized and scaled up to achieve the World Health Organization's targets to reduce physical inactivity levels by $15 \%$ by $2030^{10}$ and assist in achieving the United Nations' (UN) 2030 sustainable development goals (SDGs; Figure 1). ${ }^{11}$

Years of concerted advocacy and key documents-The Toronto Charter for Physical Activity, ${ }^{12}$ Investments that Work for Physical Activity, ${ }^{13}$ The Lancet Physical Activity Series of 2012 and 2016, and the Bangkok Declaration on Physical Activity for Global Health and Sustainable Development ${ }^{14}$-led to widespread recognition of the inactivity problem. In response to requests from countries for updated guidance, the World Health Organization launched the Global Action Plan on Physical Activity (GAPPA) 2018-2030.15 The GAPPA provides a framework of 20 effective and feasible policy actions within 4 strategic objectives to increase PA levels. Importantly, the recommended actions can contribute toward 13 of the SDGs (Figure 1). The GAPPA requires engagement from multiple stakeholders (eg, health agencies, local and national governments, nongovernmental agencies, city officials and planners, professional bodies, the media, academia, and civil society $)^{16}$ for successful implementation. 


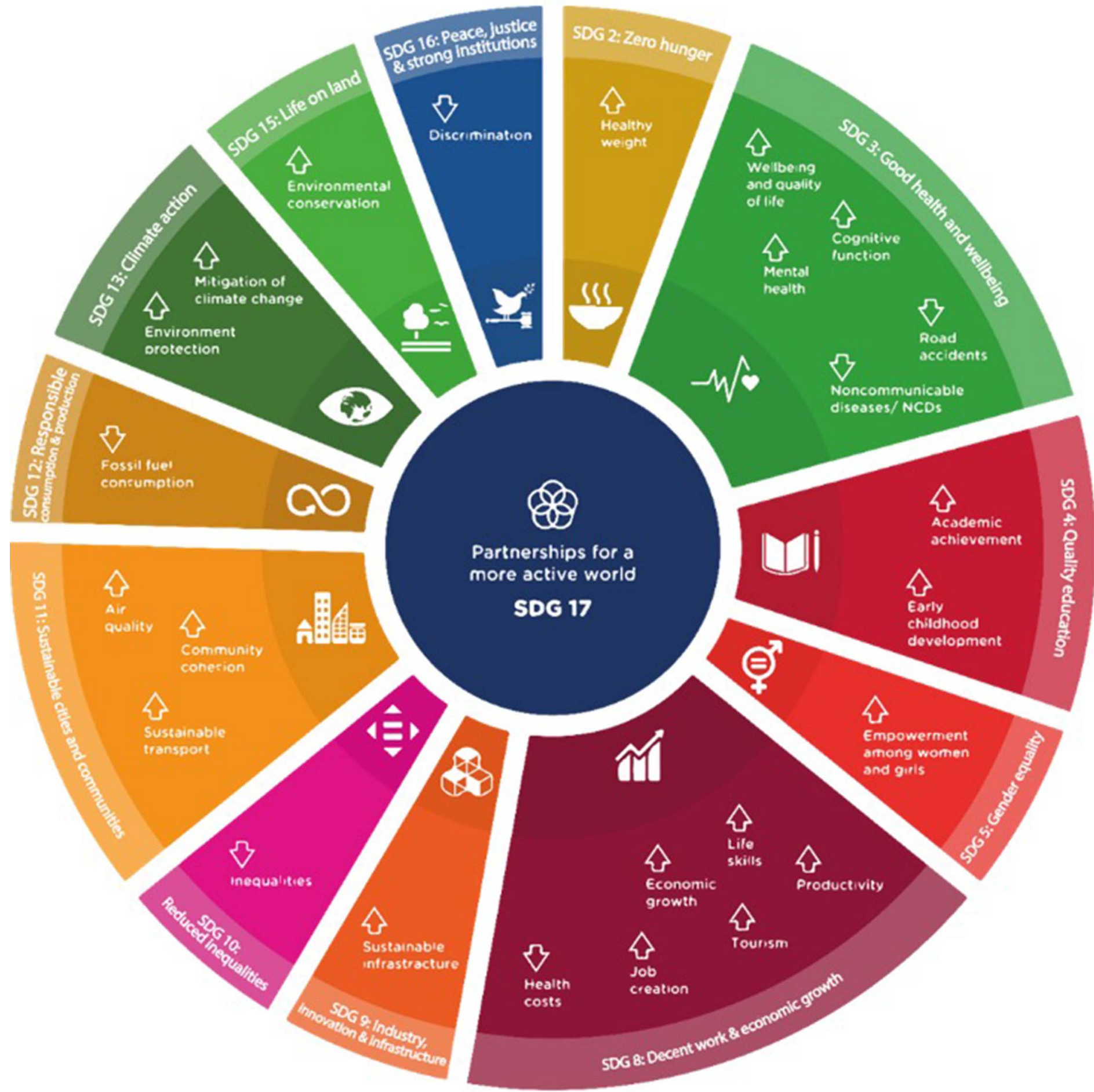

Figure 1 - Links between action on physical activity and 13 United Nations sustainable development goals (SDGs). Adapted from World Health Organization (WHO). ${ }^{15}$

Early career professionals (ECPs), including researchers, practitioners, and policymakers, can play a vital role in advocacy for the GAPPA and with aspects of the implementation of the 20 recommended actions. There are 4 overarching areas where ECPs can play a major role to help with "all people being regularly active." 15 These include (1) generation-of evidence (ie, by supplying possible solutions for decision makers to consider);
(2) dissemination - of information, materials, and GAPPA resources $^{15}$; (3) implementation-by using strategies to adopt the evidence-based actions proposed in the GAPPA and change current practices; and (4) contributing to advocacy for robust and funded national action plans on PA.

The actions within the GAPPA target different stakeholders and audiences and make use of a variety of strategies and 


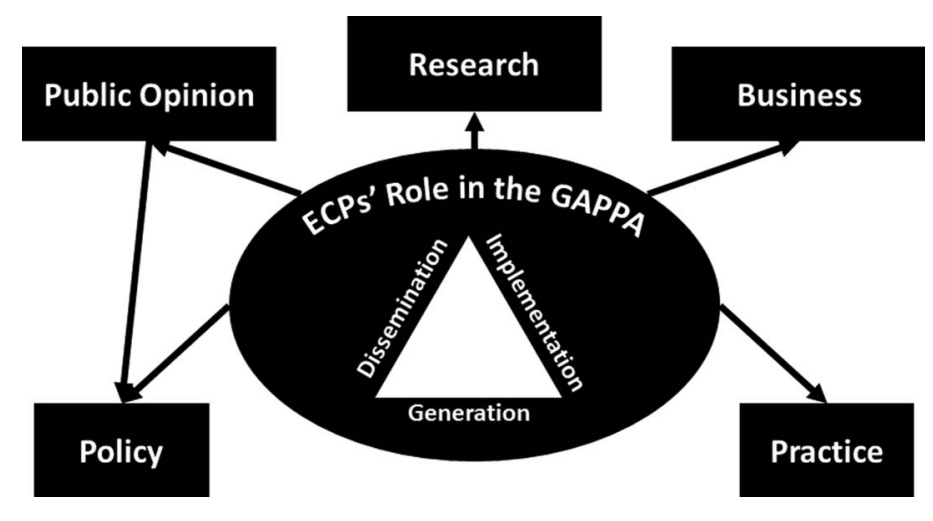

Figure 2 - Early career professionals' (ECPs) role in the Global Action Plan on Physical Activity (GAPPA). Adapted from the model of the pathways for research translation. ${ }^{17}$

communication materials. As ECPs in this area, a starting point is to become familiar with the GAPPA and understanding the actions and pathways that are available. To aid this understanding, this commentary offers suggestions and provides recommendations and examples of how ECPs can generate, disseminate, and implement the evidence and actions proposed by the GAPPA. Recommendations and examples are organized under 5 areas of focus: (1) research; (2) practice/workforce; (3) business; (4) policy; and (5) public, professional, and media opinion (Figure 2). These areas of focus originate from the recent work of Sallis, ${ }^{17}$ who put forward a model of the pathways to research translation and are informed by Shilton's ${ }^{18,19}$ model for noncommunicable disease advocacy. These models propose a variety of ways to mobilize political, media, professional, community, and organizational dimensions of advocacy to achieve the ultimate goal of translating research to practice and policy, while providing options for different actors becoming involved in research translation activities. ${ }^{17}$ From Sallis' model, our commentary provides recommendations and examples of how ECPs can generate, disseminate, and implement the evidence and actions proposed by the GAPPA. Shilton ${ }^{19}$ outlines 6 imperatives for effective advocacy and presents these in a model to inform advocacy practice. These are (1) evidence-translating and presenting evidence as urgent; (2) policy relevance-presenting PA as relevant to health and across sectors; (3) solutionsmobilizing global consensus around the key best investments; (4) partnerships/coalitions-mobilizing agencies with common objectives; (5) advocacy strategy_advocating across political, media, professional, community, and organizational dimensions; and (6) messaging-providing persuasive messages that capture the issue.

We suggest that ECPs choose their own generation, dissemination, advocacy, and implementation efforts based on the suitability of these recommendations to their roles, interests, skills, career aspirations and focus, and the timely political circumstances and opportunities in their jurisdiction.

\section{Focus Area 1-Research}

Research findings can be used to inform decision making for key stakeholders. Although not all research should be translated to practice and/or policy, relevant evidence-based solutions for decision makers to consider to help "all people being regularly active" are compiled in the GAPPA. Research, thus, plays a key role in generating, updating, and supplying feasible evidence-based solutions to aid in the reduction in physical inactivity levels. The recommendations presented in this subsection would resonate primarily with early career researchers. However, the list contains references to "decision makers" and "stakeholders," deeming some of the recommendations relevant to early career practitioners and policymakers. The ways for ECPs to contribute to the research focus include:

- Publishing research in the basic, clinical, and applied sciences of PA and health.

- Conducting transdisciplinary research with transport, education, urban planners, and other professionals (ie, linking to the UN SDGs and making findings more relevant to decision markers).

- Evaluating interventions comprehensively (ie, including formative, process, and summative evaluations), along with examining the barriers and facilitators to implementation, thereby identifying effective interventions and a clear understanding of scalability. ${ }^{20}$

- Disseminating research through national and international conferences, generating awareness, and building research networks. Consider alternative avenues to traditional academic journals to communicate with stakeholders, decision makers, and practitioners, such as presentations, blogs, or public engagement events.

- Consider consumer research to demonstrate public support for PA advocacy objectives.

\section{Focus Area 2-Practice/Workforce}

There is a clear need to work with and inform practice across multiple sectors. PA promotion can inform and be informed by a variety of other sectors, such as transportation, education, urban planning, tourism, architecture, climate, and academia. Moreover, there is a need to cover a range of levels of the workforce, from government to grassroots delivery. ECPs can contribute to the practice focus in multiple ways, such as:

- Joining and contributing to the work of professional societies from the behavioral medicine and/or PA and health-related fields (ie, encouraging a cross-pollination of knowledge).

- Mobilizing consensus across sectors and a common voice around priority GAPPA actions.

- Being open to informing and being informed by practice "beyond health professionals," such as transportation, education, urban planning, tourism, architecture, politics, and climate professionals.

- Promoting and advocating PA for specific groups with low levels of PA, with the aim of reducing inequalities.

- Helping to organize training for professional bodies, practitioners, and program-delivery personnel involved with the promotion of PA.

- Collaborating with key stakeholders for the development of audience-specific communication and dissemination products that summarize relevant PA evidence and actions in a suitable manner (ie, briefs).

- Supporting the translation of the GAPPA and/or other advocacy resources and products into the language(s) appropriate for different countries or regions. 


\section{Focus Area 3-Business}

In some instances, it can be useful for ECPs to have a business focus to generate, disseminate, and implement important evidence and actions. Consider the actions provided and how they may help with the advocacy of the GAPPA in the area of research, practice, or policy. The ways for ECPs to contribute to PA promotion within the business focus include:

- Assisting in changing business practices, promoting PA, and increasing health awareness. For example, advocate for business policies that promote safe and affordable opportunities to be physically active, regardless of sex, age, socioeconomic status, or beliefs (SDG 10 "reduced inequalities"). Involve industry partners in PA promotion, especially where the opportunities (eg, programs, training/education, capital investment) are provided in business settings and the outcomes are relevant to the companies involved.

- Developing and using entrepreneurial skills to contribute to organizations, where PA evidence drives effective PA promotion methods to populations.

- Seeking and applying for leadership training and roles in PA-related companies.

- Being alert to small business innovation research or knowledge transfer grants and opportunities for training, research, and evaluation within companies.

\section{Focus Area 4-Policy}

Early career professionals can play a role in the translation of evidence, knowledge, actions, and goals of the GAPPA at the policy level in their localities, regions, or countries. ECPs can aid and engage with the policy level through the following examples:

- Supporting the production of policy briefs that summarize evidence for policy actions and provide information for decision makers. Multiple levels and agents need to be considered, including governments (eg, local councils, regional, national), professional organizations, and corporations.

- Acknowledging policies published by a range of government sectors (eg, education, health, urban planning, and transport) and supporting other sectors to develop policies that support PA. For instance, developing urban and transport planning policies to provide equitable access to open spaces and places, recreational facilities, and safe infrastructure to walking and cycling. This can contribute toward sustainable transport systems for all, achieving universal access to green and public spaces and reducing the environmental impact of cities, which, in turn, can contribute toward SDG 11 "sustainable cities and communities."

- Ensuring to specify the policy relevance of your work, highlighting the important and politically relevant cobenefits of actions to increase PA. Examples of this are the inclusion of cost-effectiveness evaluations of relevant work to inform policy or the advocacy of the GAPPA actions, which can directly contribute to the UN 2030 SDGs.

- Seeking opportunities to present findings, products, and tools of your work to the relevant stakeholders at the policy level. This could be through government-led academic engagement seminars or attendance and contribution at public health conferences.
- Working collectively and engaging policymakers when selecting and designing research questions (ie, cocreation) to ensure the relevance and feasibility for real-world application.

\section{Focus Area 5-Public, Professional, and Media Opinion}

It is important to disseminate the findings of relevant work and advocate for the promotion of PA among the general public, through our professional allies and key influencers in the media. Mobilizing engagement with the public can help promote PA engagement though another pathway, although previous focus operates at more distal levels (eg, policy, business). There are a number of ways for achieving this, such as:

- Communicating findings or general information directly to the public through press releases, media events, social media platforms with the goal of indirectly affecting future policy decisions. Public opinion may have a powerful impact on policy decisions.

- Mobilizing professional consensus for advocacy actions through conferences, webinars, electronic direct mail, journals, websites and other "owned media," Twitter, LinkedIn, and other relevant platforms.

- Undertaking training to enhance the communication and media skills for disseminating your work via widely viewed media/press outlets.

- Building relationships with media/public relations/communication experts (eg, health journalists and writers, commentators, and marketing departments within organizations) to help communicate your findings in ways that the media and public find compelling.

- Communicating findings, outputs, and tools in "layman's terms" through alternative methods (eg, social media, news outlets, blogs) with the goal of building support for specific policies.

- Seeking partnership with advocacy organizations and individuals that have expertise in communicating research-based or health promotion messages across diverse channels (eg, noncommunicable disease alliance, International Union for Health Promotion and Education, Sustrans).

- Mobilizing the public to advocate for programs, supportive environments, and environmental changes in their communities through petitions, Facebook, mass participation events, and meetings with their local political representatives.

\section{What Are the Next Steps?}

It is advised that ECPs use available professional development opportunities to help understand the GAPPA and how best to advocate it through multiple areas of focus. This might include identifying an advocacy mentor through relevant societies, such as the International Society for Physical Activity and Health. The suggestions provided in this commentary can be utilized by ECPs, depending on their role, experience, and area of focus, to support effective advocacy, dissemination, and implementation of the GAPPA actions. There is a role for everyone in advocacy processes.

To support this professional community development, the Early Career Network of the International Society for Physical Activity and Health will undertake an assessment of ECPs' needs to better understand the GAPPA and which support and models may 
be necessary to facilitate its advocacy. This will be followed by a workshop that will address the queries derived from the needs assessment. As a network with the capacity to provide professional community development, we aim for these future activities to increase understanding, in turn, leading to effective advocacy, dissemination, and implementation of the GAPPA actions in robust and funded national PA action plans across the world. Our collective advocacy can deliver substantial return on investment in achieving the goal of "more active people for a healthier world."

\section{Conclusions}

Action is needed from multiple stakeholders operating at multiple levels, with ECPs having a critical role in supporting the implementation of the GAPPA at the national and local levels. The actions highlighted in this commentary can support ECPs in advocating for PA and translating the GAPPA into practice. Through our collective action, let's ensure that ECPs play their role in contributing to the achievement of the World Health Organization target for reducing physical inactivity by $15 \%$ by 2030 .

\section{Acknowledgments}

The authors thank Dr Fiona Bull and Dr Karen Milton for their input on earlier drafts of this commentary. We acknowledge the support of the International Society for Physical Activity and Health (ISPAH). All authors declare they have no relevant financial interests related to the present article.

\section{References}

1. Lee IM, Shiroma EJ, Lobelo F, et al. Effect of physical inactivity on major non-communicable diseases worldwide: an analysis of burden of disease and life expectancy. Lancet. 2012;380(9838):219-229. PubMed ID: 22818936 doi:10.1016/S0140-6736(12)61031-9

2. Ding D, Lawson KD, Kolbe-Alexander TL, et al. The economic burden of physical inactivity: a global analysis of major non-communicable diseases. Lancet. 2016;388(10051):1311-1324. PubMed ID: 27475266 doi:10.1016/S0140-6736(16)30383-X

3. Heath GW, Parra DC, Sarmiento OL, et al. Evidence-based intervention in physical activity: lessons from around the world. Lancet. 2012;380(9838):272-281. PubMed ID: 22818939 doi:10.1016/ S0140-6736(12)60816-2

4. Klepac Pogrmilovic B, O'sullivan G, Milton K, et al. A global systematic scoping review of studies analysing indicators, development, and content of national-level physical activity and sedentary behaviour policies. Int J Behav Nutr Phys Act. 2018;15(1):123. PubMed ID: 30486826 doi:10.1186/s12966-018-0742-9

5. Sallis JF, Bull F, Guthold R, et al. Progress in physical activity over the Olympic quadrennium. Lancet. 2016;388(10051):1325-1336. PubMed ID: 27475270 doi:10.1016/S0140-6736(16)30581-5

6. Guthold R, Stevens GA, Riley LM, Bull FC. Worldwide trends in insufficient physical activity from 2001 to 2016: a pooled analysis of
358 population-based surveys with 1.9 million participants. Lancet Glob Health. 2018;6(10):E1077-E1086. PubMed ID: 30193830 doi:10.1016/S2214-109X(18)30357-7

7. Ng SW, Popkin BM. Time use and physical activity: a shift away from movement across the globe. Obes Rev. 2012;13(8):659-680. PubMed ID: 22694051 doi:10.1111/j.1467-789X.2011.00982.x

8. Brownson RC, Royer C, Ewing R, McBride TD. Researchers and policymakers: travelers in parallel universes. Am J Prev Med. 2006; 30(2):164-172. PubMed ID: 16459216 doi:10.1016/j.amepre.2005. 10.004

9. Chalmers I, Bracken MB, Djulbegovic B, et al. How to increase value and reduce waste when research priorities are set. Lancet. 2014; 383(9912):156-165. doi:10.1016/S0140-6736(13)62229-1

10. Foster C, Shilton T, Westerman L, Varney J, Bull F. World Health Organisation to develop global action plan to promote physical activity: time for action. Br J Sports Med. 2018;52(8):484-485. PubMed ID: 28724712 doi:10.1136/bjsports-2017-098070

11. United Nations. Transforming Our World: The 2030 Agenda for Sustainable Development. 2015. https://sustainabledevelopment.un. org/content/documents/21252030\%20Agenda\%20for\%20Sustainable \%20Development\%20web.pdf. Published 2015. Accessed December 10, 2018.

12. Global Advocacy Council for Physical Activity, International Society for Physical Activity and Health. The Toronto Charter for Physical Activity: A Global Call to Action. 2010. https://ncdalliance.org/sites/ default/files/resource_files/torontocharter-eng-20may2010.pdf. Accessed December 1, 2016.

13. Global Advocacy for Physical Activity (GAPA) the Advocacy Council of the International Society for Physical Activity and Health (ISPAH). NCD Prevention: Investments that Work for Physical Activity. 2012. https://bjsm.bmj.com/content/bjsports/46/10/709. full.pdf. Accessed December 1, 2016.

14. International Society for Physical Activity and Health. The Bangkok Declaration on Physical Activity for Global Health and Sustainable Development. 2016. http://www.webcitation.org/6mWsbWh5W. Accessed December 2, 2016.

15. World Health Organization (WHO). Global Action Plan for Physical Activity: More Active People for a Healthier World. Geneva: World Health Organization. 2018.

16. Murray A, Foster C, Stamatakis E. Let's share, help deliver and sustain the WHO global action plan on physical activity. Br J Sports Med. 2019;53(13):794-796. PubMed ID: 30626596 doi:10.1136/ bjsports-2018-100099

17. Sallis JF. Pathways for translating behavioral medicine research to practice and policy. Transl Behav Med. 2018. PubMed ID: 30508151 doi:10.1093/tbm/iby 103

18. Shilton T. Creating and making the case: global advocacy for physical activity. J Phys Act Health. 2008;5(6):765-776. PubMed ID: 19164814 doi:10.1123/jpah.5.6.765

19. Shilton T. Advocacy for non-communicable disease preventionbuilding capacity in Japan. Jpn J Health Educ Promot. 2016;24(2): 102-117.

20. Indig D, Lee K, Grunseit A, Milat A, Bauman A. Pathways for scaling up public health interventions. BMC Public Health. 2017;18(1):68. PubMed ID: 28764785 doi:10.1186/s12889-017-4572-5 